\title{
Analyzing the impact of Artificial Intelligence in Online business Intelligence
}

\author{
Tanmayee Tushar Parbat ${ }^{1}$, Rohan Benhal ${ }^{2}$, Honey Jain ${ }^{1}$, Dr. Vinayak Musale ${ }^{3}$ \\ 'B.E IT, Dr. Vishwanath Karad MIT World Peace University, Pune, Maharashtra, India \\ ${ }_{2}^{2 B B A}$ IT, Dr. Vishwanath Karad MIT World Peace University, Pune, Maharashtra, India \\ ${ }^{3}$ Dr. Vishwanath Karad MIT World Peace University, Pune, Maharashtra, India
}

\begin{abstract}
Article Info

Volume 7, Issue 6

Page Number: 269-275

Publication Issue :

November-December-2021

Article History

Accepted : 05 Dec 2021

Published : 15 Dec 2021

The replacement of traditional shopping fashion by the varied modes of online shopping in real-time. Due to traditional shopping, most of them are becoming into real feel about the merchandise whichever they buy. The merchandise features are going to be manually realized by the consumers whereas in online shopping all the consumers believe the descriptive summary of the products and therefore the various factors supported the sold historical data. Now a day's modern shopping method is moving gradually towards hitting a greater number of consumers. Here recommendation system playing an important role in suggesting the merchandise by considering the sooner records and increasing the demand. Many of the consumers are attracted by factors like deals on an item, rating, review, and price of the merchandise. Through these factors, most of the consumers are interested in taking online shopping rather than traditional shopping methods. For suggesting the products to consumers, many sorts of recommendation algorithms are applied using machine learning and deep learning technology to coach the system automatically by observing the customer behavior patterns. But the believing factors of the merchandise are going to be forged some time; in such cases, consumers aren't satisfied with their expectations. the general survey of this paper will address the research gap and opportunities with the advice system.

Keywords :- Recommendation System, Bigdata, Collaborative Filtering, Machine Learning, Deep Learning, CRM, Emotional Intelligence, Influence Maintenance, Blockchain, Deep Recommender System
\end{abstract}

\section{INTRODUCTION}

Due to the advancement in online shopping, the recommender system concept has been widely applied by many of the e- commerce sites. the last word role of this technology is to research the item sales history, user purchase record and lots of of the implicit factors like liking, adding to list, 
adding to cart, bookmarking, trying to find deals that are utilized to offer a recommendation of an item to the customer. Basiliyos Tilahun Betru et.al[1] address the varied issues with the normal recommendation systems like contentbased recommendation systems, collaborative filtering, Hybrid recommendation. of these were analyzed with the deep learning technology to assess the accuracy of the advice system. The content-based recommendation techniques to aim to suggest the objects that are like those a specific user has adored earlier. Collaborative filtering may be a technique of making automated projections regarding the pursuits of a private user by gathering choices or taste data from several users. The challenge of collaborative filtering is to forecast how well a user goes to love a specific item that he has not evaluated a series of ancient preference judgments for a community of clients. CF might be administered in two ways, like itembased CF and user based CF by using two sorts of algorithms like model-based and memory- based algorithms.

\section{LITERATURE REVIEW}

Ahmed Doha et al. [2], showed that the behavioral intention towards social commerce sites and behaviors that are most ordinarily done by the consumers. Supported the buyer behavioral pattern consumers are often drawn to social commerce primarily for the pursuit of social values. The addressed research model of the works has been utilizing the three perspectives like utilitarian, economic and social perspective. The obtained results of their work are advancing a social view, which is hedonic in nature and results confirm all three perspectives.

Timur Osadchiy et al. [3], started their research work with a recommendation procedure based upon techniques like content-based and collaborative filtering. the appliance is taken to demonstrate their work on the food recommender system. They implemented recommender algorithms based upon an inherent social graph, association rule and analyzing pairwise association. Their evaluation among these three methods the pairwise association rules play superior on the required dietary recall system. pairwise association rules (PAR) suggest foods that are expected to be noticed with either of IF in pairs. Throughout the training phase PAR for every noted food $f$ calculates the amount OD [f] of foods that include that food.

Ngai et al. [4], conducted a survey on various applications of knowledge mining methods specific to Customer Relationship Management i.e., CRM. It includes a series of procedures and facilitating systems endorsing a company's decide to develop profitable and long-term relationships with certain customers. They addressed the research field of client retention and have received the foremost scientific study within the spotlight. With the more findings of limitations across their survey, they concluded that policy creators need to both maintain important customers and improve the lifelong value of the client. By itself, customer development and retention are both essential to providing a pleasing and long- term relationship with their clients.

Rajarajeshwari et al. [5], applied deep learning techniques to try to to sentiment analysis in social applications. They applied their techniques to the movie review platform. it's been taken by six phases of labor by them. Initially, they were tried with the linear machine learning algorithm to make a deep learning-based sentiment classifier utilizing a word embeddings paradigm. The developed classifier functions as a baseline to be compared to the subsequent results. Next thereto, they worked on two ensemble methods which combined their baseline classifier with other side 
classifiers utilized extensively in Sentiment Analysis. By the third model, they figured out on two prototypes that combines both the superficial and deep characteristics to mix information from multiple sources. As a fourth model, they establish a taxonomy for categorizing the varied models found in their literature. Fifth, they conducted multiple trials to match the accomplishment of these models with the deep learning standard. They analyzed the performance of the system by following the F1 measure technique.

Ivens Portugal et al. [6], conducted a literature review on various machine learning algorithms so as to use machine learning algorithms in recommender system applications. They addressed the adopted machine learning algorithms in big data technologies, identified the kinds of algorithms and their application domain, and analyzed both main and alternative performance metrics.

Weihua $\mathrm{Li}$ et al. [7], created an agent-based influence maintenance standard which will choose prominent nodes counting on this situation in dynamic social networks several times. They identified the multiple seeds to take care of the long-term influence to support and enhance business development. they need experimented with the work with wiki-vote, Email-Enron, and Ego-Facebook and demonstrated that the seed selection algorithm TIH is adept at providing longterm influence efficiently.

Zeshan Aslam Khan et al. [8], they designed a system of de-noising autoencoder-based recommender system (UT CDAE) that model's user rating trend when detecting the top-N recommendation of a user. UT CDAE technique produced outperformed other denoising autoencoder based techniques.
Shaozhong Zhang et al. [9], worked on customer suggestions and sentiments in E-commerce systems. The key parameter they need taken as trust that has been divided into two forms like propagation of trust and express trust, which characterizes a trust relationship among two people. The express trust has been identified from sentiment similarity with an entity - sentiment word pair mining technique for a resemblance feature extraction. The propagation of trust are often computed as per the transitivity features. Such two aspects might be combined utilized to research the sentiment express trust relationship. Also, they address the problems in handling sentimental review data like sparse review comments gives complexity to handle, difficulties behind the calculation of the degree of trust supported various parameters a few product and incorporating the temporal factors to capture the dynamic changes in similarity among the users of the similar product or different product.

Jia et al. [10], the key talk about their works is all about the reviews and ratings given by the customer. The comparative analysis says that the mobile reviews are greater than the PC reviews similarly the mobile ratings are above PC ratings. it's been projecting the ups and downs in review rating counts supported the higher/lower quality of the products. They need conducted the two folds of contributions. it's consolidated the connection among online word of mouth attributes and mouth channels. Followed by their system address the restrictions like working with a smaller number of economic data sets and R2 (Reviews and Ratings) is extremely low altogether models.

Shangping Wang et al. [11], fairness of payment and audit ability of an asset is that the main factor focused by the team. They resolved the challenges behind the asset distribution system. They defined the protocols for the secured transaction and 
tracking the asset delivery with the assistance of three different sorts of smart contracts such as merchants, consumers, and logistics companies. The system behavioral diagram projects the blockchain policy they need introduced with their system. With the blockchain and ethereum, they recorded the transaction during a network preceded with a timestamp block.

Scholta et al. [12], handled the business logic chain-powered smart contracts to make sure auditability, integrity, and transparency. Initial work concentrates on creating a companylevel domain knowledge graph expert for a recommendation, it might be expanded to create a greater and greater global knowledge graph. this might be achieved through initiating larger-scale crowdsourcing within several SMEs or the business-to-business alliance, which can be implemented on the consortium blockchain. With the refining and rising efficiency of the knowledge graph, the implemented system produces better performance. Then the correction of the knowledge graph is now accomplished just by reversal voting.

Helo et al. [13], implemented a cache system namely a one-stop shop to no-stop shop. the matter has been addressed as improving the design of forms to work out the only window contact during a one-stop shop, proactively initiating the services by themselves. With this, the early-stage model has been extended further to receive services from government bodies. They proved the efficiency and effectiveness of the govt services are improved with one-stop shopping. With an equivalent three- dimensional data, the model has been applied to supply friction- free delivery of state services. They introduced the research challenges in highly entrusting relationships among governments and citizens and government.
Da Coasta et al. [14], defined an answer for Realtime supply chain with the assistance of blockchain design for project deliveries with tracking and tracing of products. The cloud-based web portal has been implemented for real-time tracing and tracking of logistics and provides chain. The designed portal has combined the blockchain, IoT, and RFID technological knowledge into an embedded real-time view. Hence an immutable chain of a transaction is feasible with blockchain.

\section{COMPARATIVE ANALYSIS}

Even though various sorts of algorithms like within the above table are wont to resolve the issues of cold start, sparsity, user trust factor and accuracy prediction there are certain limitations that are found during this survey. With these shortcomings, there are possibilities to increase the research work to seek out a far better solution to beat the limitation. There are many scopes to proceed with the research in e-commerce platforms specific to consumer satisfaction level and applying emotional intelligence to live the satisfaction level.

Apart from the main references, we've conducted a web survey among young generation people with a group of questionnaires about India's various ecommerce websites which are frequently employed by most consumers. As we categorized the info supported different age groups. Various consumers face several issues with their regular ecommerce sites and hesitating to deal with the issues globally. With this survey, the buyer is asked to answer a number of the questions as presented in tabular format. the web survey has been conducted with the 850 people and therefore the summary of every question has projected below. 


\begin{tabular}{|c|c|}
\hline \multicolumn{2}{|c|}{ 1. Are you an online shopper? } \\
\hline Yes & No \\
\hline $75.6 \%$ & $24.4 \%$ \\
\hline
\end{tabular}

2. What is most important
to you when shopping
online?
Speed $-7.1 \%$
Ease of use $-15.3 \%$
Reliability $-18.8 \%$
Price $-49.4 \%$
Free Shipping $-9.4 \%$

3. What is your opinion about classifying customer profiles like prime not prime?

I'm a Prime 22.1\%

Partiality $-48.8 \%$

I hate prime $-29.1 \%$

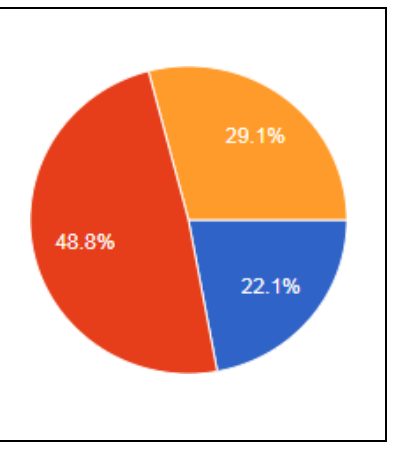

\begin{tabular}{|l|l|}
\hline 4. In what way you have & \\
been attracted by the & \\
site? & \\
\hline Through Friends $-12.6 \%$ & \\
TV Ads $-9.2 \%$ & \\
Offer Deals $-70.1 \%$ & \\
EMI $-8 \%$ & \\
\hline
\end{tabular}

\begin{tabular}{|l|l|}
\hline $\begin{array}{l}\text { 5. Which kind of } \\
\text { confidence factor that you } \\
\text { are considering before } \\
\text { placing the order? }\end{array}$ & \\
\hline Ratings $-19.1 \%$ & \\
Reviews $-56.2 \%$ & \\
Images $-6.7 \%$ & \\
Videos $-1.1 \%$ & \\
Product Descriptions $-9 \%$ & \\
\hline
\end{tabular}

6. Do you believe the reviews and ratings given
to the products are explicitly given by the
customer?
\begin{tabular}{|l|l|}
\hline Yes & No \\
\hline $77.5 \%$ & $22.5 \%$ \\
\hline
\end{tabular}

\begin{tabular}{|c|c|}
\hline \multicolumn{2}{|c|}{$\begin{array}{l}\text { 7. Will you buy products that are recommended } \\
\text { by the site based on your historical purchase } \\
\text { data? }\end{array}$} \\
\hline Yes & No \\
\hline $41.6 \%$ & $58.4 \%$ \\
\hline
\end{tabular}

$\begin{aligned} & \text { 8. Do you think that every customer purchase } \\
& \text { record is confidential with the site? }\end{aligned}$
\begin{tabular}{|l|l|}
\hline Yes & No \\
\hline $57.3 \%$ & $42.7 \%$ \\
\hline
\end{tabular}

According to the age groups of 21-25 and 26-30 are the people that are buying the products online within the most frequent manner. The results of the survey raises the questions and addresses the research opportunities with the varied belief factors that are considered by the consumers. the bulk of consumers are visiting the location by considering the TV ads, price of the merchandise, offer deals on products and majorly consumers reading the reviews written by the people that bought an equivalent and earliest. $77.5 \%$ of consumers believe the reviews and ratings given to the products that are explicitly given by the purchasers without validating such reviews and ratings. Only $41.6 \%$ of the consumers are buying the products supported the advice and $58.4 \%$ of consumers aren't believing the advice done by the location, so there'll be a niche to enhance the accuracy of the advice process. aside from this confidentiality of customer purchase records aren't so secure and are implicitly utilized at the rear end to line up the advice to others.

$57.3 \%$ of 
individuals aren't believing confidential factors. As review analysis says that the all age bracket of $60 \%$ of individuals are placing the order only by considering the rating and reviews of the merchandise . Our observation says that sometimes reviews and ratings are going to be predicted from similar products to beat the info sparsity problem.

\section{CONCLUSION}

In this article, we reviewed the varied sorts of traditional recommender systems which are implemented to resolve several real-time application problems. Then, a survey and critique of recommender systems are provided as a summary. supported the comparative study of various recommender systems with different applications, we will conclude with the issues and limitations faced by every consumer who are preferring to shop for things in online marts and therefore the accuracy of advice is additionally not satisfied by the $58.4 \%$ of individuals. The review also reveals that tons of improvement can still be done. thanks to the restrictions addressed during this survey, we introduces the research challenges in various applications of e-commerce recommender systems like, food ordering, online shopping, secure product delivery, movie reviews, customer tracking etc.. At last, future experiments could even be dedicated to the idea and experimentation of the obtained leads to the forthcoming solutions of recommender systems.

\section{REFERENCES}

[1] Betru, B. T., Onana, C. A., \& Batchakui, B. (2017). Deep learning methods on recommender system: A survey of state-of-theart. International Journal of Computer Applications, 162(10), 17-22.

[2] Doha, A., Elnahla, N., \& McShane, L. (2019).
Social commerce as social networking. Journal of Retailing and Consumer Services, 47, 307321.

[3] Osadchiy, T., Poliakov, I., Olivier, P., Rowland, M., \& Foster, E. (2019). Recommender system based on pairwise association rules. Expert Systems with Applications, 115, 535-542.

[4] Ngai, E. W., Xiu, L., \& Chau, D. C. (2009). Application of data mining techniques in customer relationship management: A literature review and classification. Expert systems with applications, 36(2), 2592-2602.

[5] Rajarajeshwari, K., \& Radhamani, G. (2019). A Naïve Bayes Model using Semi-Supervised Parameters for Enhancing the Performance of Text Analytics. International Journal of Advanced Networking and Applications, 10(6), 4083-4089.

[6] Portugal, I., Alencar, P., \& Cowan, D. (2018). The use of machine learning algorithms in recommender systems: A systematic review. Expert Systems with Applications, 97, 205-227.

[7] Li, W., Bai, Q., Zhang, M., \& Nguyen, T. D. (2018). Automated influence maintenance in social networks: an agent-based approach. IEEE Transactions on Knowledge and Data Engineering, 31(10), 1884-1897.

[8] Khan, Z. A., Zubair, S., Imran, K., Ahmad, R., Butt, S. A., \& Chaudhary, N. I. (2019). A New Users Rating-Trend Based Collaborative Denoising Auto-Encoder for Top-N Recommender Systems. IEEE Access, 7, 141287141310.

[9] Zhang, S., \& Zhong, H. (2019). Mining users trust from e- commerce reviews based on sentiment similarity analysis. IEEE Access, 7, 13523-13535.

[10] Jia, S. S., \& Wu, B. (2019). User Generated Information on Mobile Channels With More Concise Reviews and More Extreme Ratings. IEEE Access, 7, 83495-83503.

[11] Wang, S., Tang, X., Zhang, Y., \& Chen, J. 
Tanmayee Tushar Parbat et al Int. J. Sci. Res. Comput. Sci. Eng. Inf. Technol, November-December-2021, 7 (6) : 269-275

(2019). Auditable Protocols for Fair Payment and Physical Asset Delivery Based on Smart Contracts. IEEE Access, 7, 109439-109453.

[12] Scholta, H., Mertens, W., Kowalkiewicz, M., \& Becker, J. (2019). From one-stop shop to no-stop shop: An e- government stage model. Government Information Quarterly, 36(1), 1126.

[13] Helo, P., \& Shamsuzzoha, A. H. M. (2020). Real-time supply chain-A blockchain architecture for project deliveries. Robotics and Computer-Integrated Manufacturing, 63, 101909.

[14] Da Costa, A. F., Manzato, M. G., \& Campello, R. J. (2019). Boosting collaborative filtering with an ensemble of co- $^{-}$trained recommenders. Expert Systems with

Applications, 115, 427-441.

[15] AbdulHussien, A. A. (2017). Comparison of machine learning algorithms to classify web pages. International Journal of Advanced Computer Science and Applications (ijacsa), 8(11).

[16] Raphaeli, O., Goldstein, A., \& Fink, L. (2017). Analyzing online consumer behavior in mobile and PC devices: A novel web usage mining approach. Electronic commerce research and applications, 26, 1-12.

[17] Filippini, D., Alimelli, A., Di Natale, C., Paolesse, R., D'Amico, A., \& Lundström, I. (2006). Chemical sensing with familiar devices. Angewandte Chemie International Edition, 45(23), 3800-3803.

[18] Vincent, P., Larochelle, H., Bengio, Y., \& Manzagol, P. A. (2008, July). Extracting and composing robust features with denoising autoencoders. In Proceedings of the 25th international conference on Machine learning (pp. 1096- 1103).

[19] Ethereum. Accessed: Dec. 28, 2018. [Online]. Available: https://www.stateofthedapps.com/zh BTC. Accessed: Dec. 28, 2018. [Online].
Available:

https://www.

8btc.com/article/117055

[20] Forget, P., D’Amours, S., \& Frayret, J. M. (2008). Multi- behavior agent model for planning in supply chains: An application to the lumber industry. Robotics and Computer- Integrated Manufacturing, 24(5), 664-679.

[21] Su, Q., \& Chen, L. (2015). A method for discovering clusters of e-commerce interest patterns using click-stream data. electronic commerce research and applications, 14(1), 113.

[22] Wang, W., Zhang, G., \& Lu, J. (2017). Hierarchy visualization for group recommender systems. IEEE Transactions on Systems, Man, and Cybernetics: Systems, 49(6), 1152-1163.

[23] Park, H. S., \& Jun, C. H. (2009). A simple and fast algorithm for K-medoids clustering. Expert systems with applications, 36(2), 33363341.

\section{Cite this article as :}

Tanmayee Tushar Parbat, Rohan Benhal, Honey Jain, Dr. Vinayak Musale, "Analyzing the impact of Artificial Intelligence in Online business Intelligence", International Journal of Scientific Research in Computer Science, Engineering and Information Technology (IJSRCSEIT), ISSN : 2456-3307, Volume 7 Issue 6, pp. 269-275, November-December 2021. Journal URL : https://ijsrcseit.com/CSEIT217674 